\title{
CIRCULANT $L$-ENSEMBLES IN THE THERMODYNAMIC LIMIT
}

\author{
PETER J. FORRESTER
}

\begin{abstract}
L$-ensembles are a class of determinantal point processes which can be viewed as a statistical mechanical systems in the grand canonical ensemble. Circulant $L$-ensembles are the subclass which are locally translationally invariant and furthermore subject to periodic boundary conditions. Existing theory can very simply be specialised to this setting, allowing for the derivation of formulas for the system pressure, and the correlation kernel, in the thermodynamic limit. For a one-dimensional domain, this is possible when the circulant matrix is both real symmetric, or complex Hermitian. The special case of the former having a Gaussian functional form for the entries is shown to correspond to free fermions at finite temperature, and be generalisable to higher dimensions. A special case of the latter is shown to be the statistical mechanical model introduced by Gaudin to interpolate between Poisson and unitary symmetry statistics in random matrix theory. It is shown in all cases that the compressibility sum rule for the two-point correlation is obeyed, and the small and large distance asymptotics of the latter are considered. Also, a conjecture relating the asymptotic form of the hole probability to the pressure is verified.
\end{abstract}

\section{INTRODUCTION}

Point processes of the type familiar in statistical mechanics consist of $N$ indistinguishable particles confined to a domain $\Omega$. Let particle $j$ have coordinate $x_{j}$, and denote a configuration of the $N$-particles by $\boldsymbol{X}_{N}$. Each configuration of particles in the domain is specified by a probability density function $p^{(N, \Omega)}\left(\boldsymbol{X}_{N}\right)$. Important in both applications and for illustrative purposes is the case that $\Omega$ is an interval $[0, L]$ divided into $M$ lattice points at $\tau j / M, j=1, \ldots, M$, and where $\tau:=L / M$. Then the probability density function takes on a discrete set of values, and with the particle coordinates ordered

$$
0<x_{1} \leq x_{2} \leq \cdots \leq x_{N} \leq M
$$

is a probability,

$$
p^{(N, \Omega)}\left(\boldsymbol{X}_{N}\right)=\operatorname{Pr}\left(\boldsymbol{X}_{N}\right) .
$$

Parameterising $\Omega$ by $\boldsymbol{M}=\{1, \ldots, M\}$, and each $x_{j}$ by an integer $n_{j}$ such that $x_{j}=\tau n_{j} / M, \boldsymbol{X}_{N}$ can be regarded as a subset of $\boldsymbol{M}$ of size $N$.

Fundamental to the statistical description of point processes are the $k$-point correlation functions $\left\{\rho_{(k)}\right\}$. In the continuous setting, with no ordering of the particle coordinates assumed, these are specified in terms of the probability density function by

$$
\rho_{(k)}^{(N, \Omega)}\left(\boldsymbol{X}_{k}\right)=N(N-1) \cdots(N-k+1) \int_{\Omega} d x_{k+1} \cdots \int_{\Omega} d x_{N} p^{(N, \Omega)}\left(\boldsymbol{X}_{N}\right) .
$$

The case $k=1$ gives the particle density, with the characterising property that $\int_{a}^{b} \rho_{(1)}(x) d x$ is equal to the expected number of particles in the interval $[a, b]$. More generally the ratio

$$
\rho_{(k)}^{(N, \Omega)}\left(\boldsymbol{X}_{k}\right) / \rho_{(k-1)}^{(N, \Omega)}\left(\boldsymbol{X}_{k-1}\right)
$$


has the interpretation of corresponding to the particle density at $x_{k}$, given there are particles at $x_{1}, \ldots, x_{k-1}$. In the discrete setting, with an ordering convention such as (1.1) and $\boldsymbol{X}_{k}$ regarded as a subset, an appropriate modification of (1.3) is to define

$$
\rho_{(k)}^{(N, \boldsymbol{M})}\left(\boldsymbol{X}_{k}\right)=\operatorname{Pr}\left(\boldsymbol{X}_{k}\right)=\sum_{\boldsymbol{Y}_{N-k}:\left|\boldsymbol{X}_{k}\right|+\left|\boldsymbol{Y}_{N-k}\right|=N} p^{(N, \boldsymbol{M})}\left(\boldsymbol{X}_{k} \cup \boldsymbol{Y}_{N-k}\right) .
$$

Note the absence of the combinatorial factor $N(N-1) \cdots(N-k+1)$ which is present in (1.3). This is in keeping with subsets not recording order.

The theme of the present paper relates to the circumstance that the probability density function $p^{(N, \Omega)}$ has the particular functional form

$$
p^{(N, \Omega)}\left(\boldsymbol{X}_{N}\right)=\operatorname{det}\left[K^{(N, \Omega)}\left(x_{j}, x_{l}\right)\right]_{j, l=1}^{N}
$$

for some function $K^{(N, \Omega)}(x, y)$ referred to as the correlation kernel. Note that for repeated elements in $\boldsymbol{X}_{N}$ (or equivalently, particles at the same site), $p^{(N, \Omega)}=0$. Moreover, we want this structure to be maintained upon forming the $k$-point correlation (1.3), so that

$$
\rho_{(k)}^{(N, \Omega)}\left(\boldsymbol{X}_{k}\right)=\operatorname{det}\left[K^{(N, \Omega)}\left(x_{j}, x_{l}\right)\right]_{j, l=1}^{k},
$$

where $K^{(N, \Omega)}(x, y)$ is the same function as in (1.6). Such point processes are termed determinantal. Although this class may seem very restrictive, it has been known for some time to include a number of prominent model systems in mathematical physics. Examples include free fermion many body wave functions in quantum mechanics [1, 2]), unitary invariant random matrix ensembles $[3,4]$ ), statistical mechanics of log-potential Coulomb systems at a special coupling [5-7], statistical mechanics of certain two-dimensional lattice models [8], Dyson Brownian motion on certain matrix spaces [9], and non-intersecting random walkers [10]. As a non-exhaustive list of reviews and extended accounts of these examples and more, we reference [11-20, 51].

Reviews and extended accounts of theoretical developments of determinantal point processes, often incorporating as well many examples from mathematical physics and other settings too, are similarly numerous. Again as a non-exhaustive list we reference [14, 22-32]. Here the works $[28,29]$ place an emphasis on properties of determinantal point processes of particular relevance to machine learning and statistical inference. These are thus outside of the earlier applications in mathematical physics. Distinguishing the applications in mathematical physics is what in statistical mechanics is referred to as the thermodynamic limit - this is when the number of particles and system volume go to infinity simultaneously such that the average density is a constant. Among determinantal point processes is a special structure when the correlation kernel has a difference property $K^{(N, \Omega)}\left(x_{j}, x_{l}\right)=K^{(N, \Omega)}\left(x_{j}-x_{l}, 0\right)$. Suppose in addition that the correlation kernel is periodic of period $L$, where $L$ is the length of the interval specifying $\Omega$. Then it turns out that a formulation in what in statistical mechanics terminology is called the grand canonical ensemble — termed $L$-ensembles in the theory of determinantal point processes - allows for an analytic treatment in which the thermodynamic limit is readily computed. This working leads to the functional form for the limiting correlation kernel

$$
K^{(\infty)}(X, Y)=z \int_{-\infty}^{\infty} \frac{e^{2 \pi i(Y-X) s} \lambda^{(\infty)}(s)}{1+z \lambda^{(\infty)}(s)} d s
$$


obtained in (2.35) below. Here $z$ is the fugacity and $\lambda^{(\infty)}(s) \geq 0$ is determined by the functional form of the entries of $L^{(M)}$. The mechanism underlying this calculation is that the matrix determining the correlation kernel is circulant. A development of the consequences of a circulant structure from a viewpoint in statistical mechanics is the explicit theme of the present work.

From a technical perspective, this undertaking is straightforward: existing formulas [14] suffice for the general cases, and moreover special cases of the necessary working can already be found in the literature [33]. On the other hand, no one work logically develops circulant $L$-ensembles, nor does any single work isolate physically motivated examples from this viewpoint. Filling this gap in the literature is the contribution of the present work. In section 2 we begin by revising the formalism of $L$-ensembles, and in particular the formula for the correlation kernel in terms of a particular matrix $L^{(M)}$. When this matrix is circulant, the correlation kernel can be computed explicitly, and evaluated in certain limits. First considered is a continuum limit when the number of lattice sites goes to infinity but the interval stays fixed - this is equivalent to a grand canonical formalism defined to begin on the interval; subsection 2.5 gives a direct approach in this setting. However this does not correspond to a thermodynamic limit as the expected total number of particles is still finite. True thermodynamic limits are considered next, starting with a limit when the lattice spacing stays fixed with the number of lattice sites going to infinity, which then is an example of a lattice gas. Taking the lattice spacing to zero then specifies a statistical state defined on the whole real line. These are considered in subsection 2.4 in the case of $L^{(M)}$ being real symmetric, and in subsection 2.5 when this matrix is complex Hermitian.

Section 3 begins by showing that $L$-ensembles in the thermodynamic limit of the type considered in section 2 all obey the compressibility sum rule from the theory of fluids. This has significance in relation to the gap probability - that is the probability that a prescribed interval is free of eigenvalues, as in this setting it has previously been conjectured that the leading asymptotic form of this probability is equal to the exponential of minus of the pressure. Known asymptotics of Toeplitz operators allow this to be checked for the circulant $L$-ensembles in the thermodynamic limit. In subsection 3.4 the particular example of a real circulant $L$-ensemble with the underlying matrix having entries given by a Gaussian functional form is considered. The function $\lambda^{(\infty)}(s)$ in (2.35) is then also a Gaussian. Upon appropriate identification of parameters, (1.8) can then be identified with that for free fermions in one dimension at finite temperature,

$$
K^{(\infty)}(X, Y)=\frac{1}{2 \pi} \int_{-\infty}^{\infty} \frac{e^{i(Y-X) k}}{e^{\beta\left(k^{2}-\mu\right)}+1} d k
$$

Here $\beta$ is the inverse temperature, and $\mu$ is the chemical potential. In the following subsection, a specific example of the complex Hermitian circulant $L$-ensemble is given which can be identified with a statistical mechanical model introduced by Gaudin for application in random matrix theory. The final subsection considers a higher dimensional analogue of the Gaussian functional form, and the resulting correlation kernel is identified with that for free fermions in $d$-dimensions at finite temperature.

\section{2. $L$-ENSEMBLES}

2.1. Formalism. Consider the discrete setting specified in the opening paragraph of the Introduction, using a subset viewpoint of $\mathbf{X}_{N}$, but modified so that the value of $N$ can vary. This is 
done by specifying that for each $N=0, \ldots, M$

$$
\operatorname{Pr}\left(\boldsymbol{X}_{N}\right)=\frac{z^{N}}{\Xi^{(\boldsymbol{M})}(z)} \operatorname{det}\left[L^{(\boldsymbol{M})}\left(x_{j}, x_{l}\right)\right]_{j, l=1}^{N},
$$

where $z>0$ is a parameter, and $\Xi^{(M)}(z)$ the normalisation. Such probabilities on subsets of $\boldsymbol{M}$ are referred to as $L$-ensembles. In statistical mechanics, $\Xi^{(\boldsymbol{M})}(z)$ is referred to as the grand canonical partition function and $z$ as the fugacity. From expansion properties of the determinant it is easy to see that with $L^{(\boldsymbol{M})}:=\left[L^{(\boldsymbol{M})}(j, l)\right]_{j, l=1}^{M}$

$$
\Xi^{(M)}(z):=\sum_{N=0}^{M} z^{N} \sum_{\boldsymbol{X}_{N} \subset \boldsymbol{M}} \operatorname{det}\left[L^{(\boldsymbol{M})}\left(x_{j}, x_{l}\right)\right]_{j, l=1}^{N}=\operatorname{det}\left(\mathbb{I}_{M}+z L^{(\boldsymbol{M})}\right) .
$$

In this setting the $k$-point correlation function is defined by

$$
\rho_{(k)}^{(\boldsymbol{M})}\left(\boldsymbol{X}_{k}\right)=\sum_{s=0}^{M-k} \sum_{\substack{\boldsymbol{Y}_{s} \subset M \\\left|\mathbf{Y}_{s}\right|=s}} \operatorname{Pr}\left(\boldsymbol{X}_{k} \cup \boldsymbol{Y}_{s}\right)
$$

All $L$-ensembles are in fact determinantal point processes.

Proposition 2.1. (Macchi [2]) For an $M \times M$ matrix $J$, let $(J)_{\boldsymbol{X}_{k}}$ denote the $k \times k$ submatrix formed from the entries in the rows and columns labelled by $\boldsymbol{X}_{k}$. With this notation, we have

$$
\rho_{(k)}^{(\boldsymbol{M})}\left(\boldsymbol{X}_{k}\right)=\operatorname{det}\left(K^{(\boldsymbol{M})}\right)_{\boldsymbol{X}_{k}}
$$

where

$$
K^{(\boldsymbol{M})}=z L^{(\boldsymbol{M})}\left(\mathbb{I}_{M}+z L^{(\boldsymbol{M})}\right)^{-1} .
$$

Proof. The sum in (2.3) permits an evaluation analogous to (2.2), implying

$$
\rho_{(k)}^{(\boldsymbol{M})}\left(\boldsymbol{X}_{k}\right)=\frac{1}{\Xi^{(\boldsymbol{M})}(z)} \operatorname{det}\left(\overline{\mathbb{I}}_{M}^{\boldsymbol{X}_{k}}+z L^{(\boldsymbol{M})}\right),
$$

where $\overline{\mathbb{I}}_{M}^{\boldsymbol{X}_{k}}$ denotes the $M \times M$ identity matrix with diagonal entries $\boldsymbol{X}_{k}$ each replaced by zero. Writing $\overline{\mathbb{I}}_{M}^{\boldsymbol{X}_{k}}=\mathbb{I}_{M}-\mathbb{I}_{M}^{\boldsymbol{X}_{k}}$, substituting the determinant formula $(2.2)$ for $\Xi^{(\boldsymbol{M})}(z)$ and using the multiplicative property of the determinant shows

$$
\rho_{(k)}^{(\boldsymbol{M})}\left(\boldsymbol{X}_{k}\right)=\operatorname{det}\left(\mathbb{I}_{M}-\mathbb{I}_{M}^{\boldsymbol{X}_{k}}\left(\mathbb{I}_{M}+z L^{(\boldsymbol{M})}\right)^{-1}\right) .
$$

Introducing $K^{(M)}$ as defined by (2.5), simple manipulation of (2.7) then shows

$$
\rho_{(k)}^{(\boldsymbol{M})}\left(\boldsymbol{X}_{k}\right)=\operatorname{det}\left(\overline{\mathbb{I}}_{M}^{\boldsymbol{X}_{k}}+\mathbb{I}_{M}^{\boldsymbol{X}_{k}} K^{(\boldsymbol{M})}\right) .
$$

The result (2.4), (2.5) now follows by expansion properties of the determinant.

2.2. Toeplitz $L$-ensembles. For (2.1) to be well defined, we must have

$$
\operatorname{det}\left[L^{(\boldsymbol{M})}\left(x_{j}, x_{l}\right)\right]_{j, l=1}^{N} \geq 0
$$

Requiring too that $L^{(\boldsymbol{M})}$ as specified in (2.2) be Hermitian, we know from linear algebra that this is equivalent to $L^{(\boldsymbol{M})}$ being semi-positive definite, and thus for any $\mathbf{c}=\left(c_{1}, \ldots, c_{M}\right)$, that

$$
\mathbf{c} L^{(\boldsymbol{M})} \mathbf{c}^{\dagger} \geq 0
$$

Let us investigate $(2.10)$ in the setting that $L^{(\boldsymbol{M})}(j, l)$ has the difference property $L^{(\boldsymbol{M})}(j, l)=$ $L^{(M)}(j-l, 0)$ so that the configurations $\boldsymbol{X}_{N}$ (thinking now in the particle picture) specified by 
(2.1) are all translationally invariant. The difference property is equivalent to saying that $L^{(M)}$ is a Toeplitz matrix. Introduce $f(\zeta)$ as a generating function for the independent entries of $L^{(M)}$, so that

$$
f(\zeta)=\sum_{j=0}^{M-1} L^{(\boldsymbol{M})}(j, 0) \zeta^{j}, \quad L^{(\boldsymbol{M})}(p, 0)=\int_{0}^{1} f\left(e^{2 \pi i x}\right) e^{-2 \pi i p x} d x .
$$

Using the latter formula herein in (2.10) shows

$$
\mathbf{c} L^{(\boldsymbol{M})} \mathbf{c}^{\dagger}=\int_{0}^{1} f\left(e^{2 \pi i x}\right)\left|C\left(e^{2 \pi i x}\right)\right|^{2} d x, \quad C\left(e^{2 \pi i x}\right)=\sum_{j=0}^{M-1} c_{j} e^{2 \pi i j x} .
$$

It follows that a sufficient condition for $L^{(M)}$ to satisfy $(2.10)$ is that $f\left(e^{2 \pi i x}\right)$ be real - which is guaranteed by the assumption that $L^{(\boldsymbol{M})}$ be Hermitian, and thus $L^{(\boldsymbol{M})}(l, j)=\overline{L^{(\boldsymbol{M})}(j, l)}$ - and furthermore that $f\left(e^{2 \pi i x}\right)$ be non-negative.

Remark 2.2. 1. It is not a necessary condition that $L^{(\boldsymbol{M})}$ be Hermitian for $(2.9)$ to hold true. See [34] for examples of tridiagonal Toeplitz matrices of this type.

2. For $\kappa:=\left(\kappa_{1}, \ldots, \kappa_{n}\right)$ a partition of non-negative integers, the Schur polynomial is defined by

$$
s_{\kappa}\left(z_{1}, \ldots, z_{n}\right)=\frac{\operatorname{det}\left[z_{j}^{\kappa_{l}+n-l}\right]_{j, l=1}^{n}}{\operatorname{det}\left[z_{j}^{n-l}\right]_{j, l=1}^{n}} .
$$

This definition extends to general tuples $\kappa$. Standard manipulations used in random matrix theory (see e.g. [19, Exercises 5.4 q.1]), starting with the formula for $L^{(\boldsymbol{M})}(p, 0)$ in $(2.11)$ substituted in the LHS of (2.9) show

$$
\begin{array}{r}
\frac{1}{N !} \operatorname{det}\left[L^{(\boldsymbol{M})}\left(x_{j}, x_{l}\right)\right]_{j, l=1}^{N}=\int_{0}^{1} d t_{1} f\left(e^{2 \pi i t_{1}}\right) \cdots \int_{0}^{1} d t_{N} f\left(e^{2 \pi i t_{N}}\right)\left|s_{\kappa}\left(e^{2 \pi i t_{1}}, \ldots, e^{2 \pi i t_{N}}\right)\right|^{2} \\
\times \prod_{1 \leq j<l \leq N}\left|e^{2 \pi i t_{l}}-e^{2 \pi i t_{j}}\right|^{2}
\end{array}
$$

where $\kappa=\left(x_{1}, \ldots, x_{N}\right)$. Given that $f\left(e^{2 \pi i t}\right)$ is non-negative, this gives another way of seeing that $\operatorname{det}\left[L^{(\boldsymbol{M})}\left(x_{j}, x_{l}\right)\right]_{j, l=1}^{N}$ is non-negative.

3. The Toeplitz matrix $\left[L^{(M)}(j-l, 0)\right]_{j, l=1}^{M}$ is not a random matrix. Rather in the $L$-ensemble formalism beginning with $(2.1)$ the matrix elements $L^{(M)}(x, y)$ are a prescribed functional form. For works which do address random Toeplitz matrices, as a non-exhaustive list we draw attention to [35-39].

2.3. Real circulant $L$-ensembles. Suppose that in addition to the difference property, the entries of $L^{(M)}$ are periodic of period $M$, which is characteristic of periodic boundary conditions, or equivalently the original interval $[0, L]$ being identified with the circumference of a circle. A systematic way for the entries of $L^{(\boldsymbol{M})}$ to have this periodicity, and to have a well defined scaling limit for large $M, L$, is to measure distance as chord length, so that

$$
L^{(\boldsymbol{M})}\left(x_{j}, x_{l}\right)=L^{(\boldsymbol{M})}\left(x_{j}-x_{l}, 0\right)=g\left((L / \pi) \sin \left(\pi\left(x_{j}-x_{l}\right) / M\right)\right),
$$

for some $g(u)$ even and real valued. Note that this latter condition implies that the matrix $\left[L^{(M)}(j-\right.$ $l, 0)]_{j, l=1}^{M}$ is real symmetric. With both the difference and periodicity property of the elements, the 
matrix $L^{(\boldsymbol{M})}$ is referred to as being circulant. The significance of this extra structure is that the normalised eigenvectors of all circulant matrices are independent of $g$ in (2.15) and given by

$$
\frac{1}{\sqrt{M}} e^{-2 \pi i p_{0} / M}\left(1, e^{2 \pi i p / M}, e^{4 \pi i p / M}, \ldots, e^{2 \pi i(M-1) / M}\right)^{T}, \quad p=0, \ldots, M-1,
$$

for any integer $p_{0}$, which we take as equal to $\lfloor-M / 2\rfloor+1$. As a consequence, the normalisation $\Xi^{(M)}(z)$ and matrix elements $K^{(M)}(x, y)$ can be computed explicitly.

Proposition 2.3. In the setting of (2.15) we have

$$
\Xi^{(M)}(z)=\prod_{p=\lfloor-M / 2\rfloor+1}^{\lfloor M / 2\rfloor}\left(1+z \lambda_{p}\right), \quad \lambda_{p}=\sum_{s=\lfloor-M / 2\rfloor+1}^{\lfloor M / 2\rfloor} g(L \sin (\pi s / M)) e^{2 \pi i p s / M}=\tilde{f}\left(e^{2 \pi i p / M}\right),
$$

where $\tilde{f}(z)$ is the particular generating function for the entries (2.15)

$$
\tilde{f}(z):=\sum_{s=\lfloor-M / 2\rfloor+1}^{\lfloor M / 2\rfloor} g(L \sin (\pi s / M)) z^{s}
$$

(cf. (2.11)), and

$$
K^{(\boldsymbol{M})}(x, y)=\frac{z}{M} \sum_{p=\lfloor-M / 2\rfloor+1}^{\lfloor M / 2\rfloor} \frac{e^{2 \pi i(y-x) p / M} \lambda_{p}}{1+z \lambda_{p}} .
$$

Proof. The form of the matrix entries (2.15) and the fact that the eigenvectors are given by $(2.16)$ implies the formula for the eigenvalues $\lambda_{p}$ in (2.17), while the determinant formula in (2.2) implies the first equality therein.

Using the diagonalisation formula

$$
L^{(\boldsymbol{M})}=V^{(\boldsymbol{M})} \operatorname{diag}\left(\lambda_{1}, \ldots, \lambda_{N}\right)\left(V^{(\boldsymbol{M})}\right)^{\dagger},
$$

where the columns of $V^{(\boldsymbol{M})}$ are given by the normalised eigenvectors $\left\{\mathbf{v}_{p}\right\}$ of $L^{(\boldsymbol{M})}$, we have from (2.5) that whenever $L^{(\boldsymbol{M})}$ is Hermitian

$$
K^{(M)}(x, y)=z \sum_{p=0}^{M-1} \frac{\left(\mathbf{v}_{p}\right)^{(x)}\left(\overline{\mathbf{v}}_{p}\right)^{(y)} \lambda_{p}}{1+z \lambda_{p}} .
$$

Here $\left(\mathbf{v}_{p}\right)^{(x)}$ denotes the $x$-th component of $\mathbf{v}_{p}$ and $K^{(\boldsymbol{M})}(x, y)$ denotes the entry in row $x$, column $y$ of the matrix $K^{(\boldsymbol{M})}$. The fact that for $L^{(\boldsymbol{M})}$ a circulant matrix, the eigenvectors are given by (2.16), implies (2.19).

2.4. Large $M$ limits. In the first paragraph of the Introduction, the parameter $M$ was introduced as the number of equally spaced lattice point used to discretise a line of length $L$. Two large $M$ limits are therefore suggested. One is when $M$ and $L$ go simultaneously to infinity and the lattice spacing remains fixed. The other is when $M \rightarrow \infty$ but $L$ remains fixed, which reclaims the point process on the continuum interval $[0, L]$. We will consider each separately. 
2.4.1. $M \rightarrow \infty, L / M$ fixed.

Proposition 2.4. Let $\tau:=L / M$ denote the lattice spacing, and with $g(u)$ as in (2.15) let

$$
\tilde{f}^{(\infty)}(\zeta):=\sum_{s=-\infty}^{\infty} g(\tau s) \zeta^{s}
$$

where the sum is assumed to be well defined for $|\zeta|=1$. We have

$$
\tau \beta P^{(\tau)}:=\lim _{\substack{M \rightarrow \infty \\ \tau \text { fixed }}} \frac{1}{M} \log \Xi^{(M)}(z)=\int_{-1 / 2}^{1 / 2} \log \left(1+z \tilde{f}^{(\infty)}\left(e^{2 \pi i t}\right)\right) d t,
$$

where the notation $\tau \beta P^{(\tau)}$ relates to the interpretation of the limit in terms of the pressure from statistical mechanics, and

$$
K^{(\tau)}(x, y):=\lim _{\substack{M \rightarrow \infty \\ \tau \text { fixed }}} K^{(\boldsymbol{M})}(x, y)=z \int_{-1 / 2}^{1 / 2} \frac{e^{2 \pi i(y-x) t} \tilde{f}^{(\infty)}\left(e^{2 \pi i t}\right)}{1+z \tilde{f}^{(\infty)}\left(e^{2 \pi i t}\right)} d t .
$$

Proof. We see from the definition (2.18) that

$$
\lim _{\substack{M \rightarrow \infty \\ \tau \text { fixed }}} \tilde{f}(\zeta)=\tilde{f}^{(\infty)}(\zeta)
$$

as specified by (2.22). The stated formulas now follow by recognising the appropriate sums in Proposition 2.3 as Riemann approximations to definite integrals.

As a check, we see from (2.2), (2.17) and (2.19) that

$$
z \frac{\partial}{\partial z} \lim _{M \rightarrow \infty} \frac{1}{M} \log \Xi^{(M)}(z)=\lim _{M \rightarrow \infty} \frac{1}{M} \operatorname{Tr} K^{(M)},
$$

which requires too the validity of interchanging the derivative with the limit on the LHS. Since the RHS is equal to $\lim _{M \rightarrow \infty} K^{(\boldsymbol{M})}(x, x)$ independent of $x$ in the present setting of periodic boundary conditions, the results (2.23) and (2.24) are consistent with (2.26). Note too that this tells us that the expected number of particles per lattice site in the $M \rightarrow \infty$ limit, $\rho^{(\tau)}$ say, is given in terms of the fugacity $z$ by

$$
\rho^{(\tau)}=z \int_{-1 / 2}^{1 / 2} \frac{\tilde{f}^{(\infty)}\left(e^{2 \pi i t}\right)}{1+z \tilde{f}^{(\infty)}\left(e^{2 \pi i t}\right)} d t .
$$

In particular, since $\tilde{f}^{(\infty)}(z) \geq 0$ for $|z|=1$, the requirement that $0 \leq \rho^{(\tau)} \leq 1$ is evident.

2.4.2. $M \rightarrow \infty, L$ fixed, followed by $L \rightarrow \infty$. Starting with $L$ fixed we must have that the lattice labels $x, y$ are also suitably scaled to correspond to points in the interval, $L x / M \rightarrow X, L y / M \rightarrow$ $Y$, and furthermore $z$ must be scaled $z \mapsto L z / M$ to allow for the sums in (1.5) to correspond to Riemann integrals. The functional form (2.15) is again appropriate, but no longer with any reference to $M$, so we define

$$
L^{(L)}(X, Y)=g((L / \pi) \sin (\pi(X-Y) / L)) .
$$

Proposition 2.5. Let $g(u)$ be as in (2.28), and suppose furthermore that it be integrable on $(-L / \pi, L / \pi)$. We have

$$
\log \Xi^{(L)}(z):=\lim _{\substack{M \rightarrow \infty \\ L \text { fixed }}} \log \Xi^{(M)}(L z / M)=\sum_{p=-\infty}^{\infty} \log \left(1+z \lambda_{p}^{(L)}\right),
$$


and

$$
K^{(L)}(X, Y):=\left.\lim _{\substack{M \rightarrow \infty \\ L \text { fixed }}}(M / L) K^{(M)}(M X / L, M Y / L)\right|_{z \mapsto L z / M}=\frac{z}{L} \sum_{p=-\infty}^{\infty} \frac{e^{2 \pi i(Y-X) p / L} \lambda_{p}^{(L)}}{1+z \lambda_{p}^{(L)}},
$$

where

$$
\lambda_{p}^{(L)}=\lim _{\substack{M \rightarrow \infty \\ L \text { fixed }}} \frac{L}{M} \lambda_{p}=L \int_{-1 / 2}^{1 / 2} g\left(\frac{L}{\pi} \sin \pi t\right) e^{2 \pi i p t} d t .
$$

Proof. These formulas follow from (2.17) and (2.19), by viewing (2.18) as a Riemann sum and thus

$$
\lim _{\substack{M \rightarrow \infty \\ L \text { fixed }}} \frac{L}{M} \tilde{f}\left(e^{2 \pi i p / M}\right)=L \int_{-1 / 2}^{1 / 2} g\left(\frac{L}{\pi} \sin \pi t\right) e^{2 \pi i p t} d t
$$

which corresponds to $(2.31)$.

After changing variables $t \mapsto t / L$ in (2.31), the large $L$ limit of the quantities in Proposition 2.5 are almost immediate, with the sums therein recognised as Riemann approximations to definite integrals.

Proposition 2.6. Let

$$
\lambda^{(\infty)}(s)=\int_{-\infty}^{\infty} g(t) e^{2 \pi i s t} d t
$$

We have

$$
\beta P=\lim _{L \rightarrow \infty} \frac{1}{L} \log \Xi^{(L)}(z)=\int_{-\infty}^{\infty} \log \left(1+z \lambda^{(\infty)}(s)\right) d s
$$

and

$$
K^{(\infty)}(X, Y):=\lim _{L \rightarrow \infty} K^{(L)}(X, Y)=z \int_{-\infty}^{\infty} \frac{e^{2 \pi i(Y-X) s} \lambda^{(\infty)}(s)}{1+z \lambda^{(\infty)}(s)} d s .
$$

Remark 2.7. We see from (2.23) and (2.22) that

$$
\lim _{\tau \rightarrow 0^{+}} \beta P^{(\tau)}=\int_{-\infty}^{\infty} \log \left(1+z \lambda^{(\infty)}(s)\right) d s,
$$

thus reclaiming (2.34), and from (2.24) and (2.22) that

$$
\left.\lim _{\tau \rightarrow 0^{+}} \frac{1}{\tau} K^{(\tau)}(X / \tau, Y / \tau)\right|_{z \mapsto \tau z}=z \int_{-\infty}^{\infty} \frac{e^{2 \pi i(Y-X) s} \lambda^{(\infty)}(s)}{1+z \lambda^{(\infty)}(s)} d s,
$$

thus reclaiming (2.35). Hence, with the functional form (2.15) for the matrix elements of $L^{(M)}$, the results for the continuum can be reclaimed from the results for the lattice, upon taking the lattice spacing to zero.

2.5. Complex circulant $L$-ensembles. In the interests of brevity, we will restrict attention in this circumstance to the setting of particles on the continuous segment $[0, L]$ in periodic boundary conditions, which can be thought of as the circumference of a circle. Measuring distance as chord length, a functional form giving rise to a complex Hermitian circulant integral operator (no longer a matrix since the domain is a continuum) is

$$
L^{(L)}(X, Y)=i h((L / \pi) \sin (\pi(X-Y+2 i \epsilon) / L)), \quad \epsilon>0,
$$


with $h(u)$ odd; cf.(2.15). Note that this latter requirement implies $\overline{L^{(L)}(X, Y)}=L^{(L)}(Y, X)$ and thus $L^{(L)}$ is a Hermitian matrix. The corresponding probability density for a configuration $\boldsymbol{X}_{N}$ is

$$
p\left(\boldsymbol{X}_{N}\right)=\frac{z^{N}}{\Xi^{(L)}(z)} \operatorname{det}\left[L^{(L)}\left(X_{j}, X_{k}\right)\right]_{j, k=1}^{N}
$$

(cf. (2.1)). Here

$$
\Xi^{(L)}(z)=\operatorname{det}(\mathbb{I}+z \mathbb{L}),
$$

where $\mathbb{L}$ is the integral operator on $[0, L]$ with kernel (2.38); the determinant can be specified as the product over the eigenvalues.

Working directly in the continuum setting, the appropriate generalisation of (2.2) is

$$
\Xi^{(L)}(z)=\prod_{p=-\infty}^{\infty}\left(1+z \lambda_{p}\right)
$$

while the appropriate generalisation of (2.21) is

$$
K^{(L)}(X, Y)=z \sum_{p=-\infty}^{\infty} \frac{v_{p}(X) \bar{v}_{p}(Y) \lambda_{p}}{1+z \lambda_{p}} .
$$

Here $\left\{\lambda_{p}\right\}$ and $\left\{v_{p}(X)\right\}$ are the eigenvalues and eigenfunctions of the integral operator $\mathbb{L}$ on $[-L / 2, L / 2]$ (the periodicity in shifts by $L$ of $(2.38)$ has been used to translate the interval) with kernel (2.38). Thus

$$
\mathbb{L}[f](X)=i \int_{-L / 2}^{L / 2} h((L / \pi) \sin (\pi(X-Y+2 i \epsilon) / L)) f(Y) d Y .
$$

The normalised eigenfunctions of $\mathbb{L}$ are

$$
v_{p}(X)=\frac{1}{\sqrt{L}} e^{2 \pi i p X / L}, \quad p \in \mathbb{Z},
$$

which allows for (2.41) and (2.42) to be made explicit, and moreover for computation of the limit $L \rightarrow \infty$.

Proposition 2.8. The formulas (2.41) and (2.42) hold with $v_{p}(x)$ given by (2.44) and

$$
\lambda_{p}=i \int_{-L / 2}^{L / 2} h((L / \pi) \sin (\pi(-Y+2 i \epsilon) / L)) e^{2 \pi i p Y / L} d Y .
$$

Furthermore, with

$$
\lambda^{(\epsilon, \infty)}(s):=i \int_{-\infty}^{\infty} h(-Y+2 i \epsilon) e^{2 \pi i s Y} d Y
$$

replacing $\lambda^{(\infty)}(s)$, the formulas (2.34) and (2.35) again hold.

\section{Properties And examples}

3.1. Compressibility sum rule. In this section, in distinction to the subset viewpoint of a configuration $\boldsymbol{X}_{N}$ used in Section 2.1, it is convenient to consider $\boldsymbol{X}_{N}$ as an $N$-tuple, not to require an ordering of the particles such as in (1.1), and to consider the domain as continuous. The grand canonical ensemble formalism in this setting specifies that the probability density function for there being $N$ particles in configuration $\boldsymbol{X}_{N}$ be given by

$$
p^{(N, \Omega)}\left(\boldsymbol{X}_{N}\right)=\frac{z^{N}}{\Xi^{(\Omega)}(z)} \operatorname{det}\left[L^{(\Omega)}\left(x_{j}, x_{l}\right)\right]_{j, l=1}^{N}
$$


where

$$
\Xi^{(\Omega)}(z)=1+\sum_{N=1}^{\infty} z^{N} \int_{\Omega} d x_{1} \cdots \int_{\Omega} d x_{N} \operatorname{det}\left[L^{(\Omega)}\left(x_{j}, x_{l}\right)\right]_{j, l=1}^{N}
$$

cf. (2.1) and (2.2). The corresponding $k$-point correlation function is given by

$$
\begin{aligned}
\rho_{(k)}^{(\Omega)}\left(\boldsymbol{X}_{k}\right)=z^{k}\left(k ! p^{(k, \Omega)}\right. & \left(\boldsymbol{X}_{k}\right) \\
& \left.+\sum_{n=1}^{\infty} z^{n}(n+k) \cdots(n+1) \int_{\Omega} d y_{1} \cdots \int_{\Omega} d y_{n} p^{(k+n, \Omega)}\left(\boldsymbol{X}_{k} \cup \boldsymbol{Y}_{n}\right)\right) .
\end{aligned}
$$

Suppose furthermore that the system is translationally invariant. Then

$$
\int_{\Omega} d x_{2} \rho_{(2)}^{(\Omega)}\left(x_{1}, x_{2}\right)=\frac{1}{|\Omega|} \int_{\Omega} d x_{1} \int_{\Omega} d x_{2} \rho_{(2)}^{(\Omega)}\left(x_{1}, x_{2}\right)=\frac{1}{|\Omega|} \frac{1}{\Xi^{(\Omega)}(z)} z \frac{\partial^{2}}{\partial z^{2}} \Xi^{(\Omega)}(z) .
$$

Simple manipulation, using the fact that for a translationally invariant system

$$
\rho_{(1)}^{(\Omega)}(x)=\frac{1}{|\Omega|} \frac{\partial}{\partial z} \log \Xi^{(\Omega)}(z),
$$

shows from this that

$$
\int_{\Omega} d x_{2}\left(\rho_{(2)}^{(\Omega)}\left(x_{1}, x_{2}\right)-\rho_{(1)}^{(\Omega)}\left(x_{1}\right) \rho_{(1)}^{(\Omega)}\left(x_{2}\right)+\delta\left(x_{1}-x_{2}\right) \rho_{(1)}^{(\Omega)}\left(x_{2}\right)\right)=\frac{1}{|\Omega|}\left(z \frac{\partial}{\partial z}\right)^{2} \log \Xi^{(\Omega)}(z) .
$$

Taking the limit $|\Omega| \rightarrow \infty$, assuming the limit operation can be taken inside the integral on the LHS, and the derivative operation on the RHS, then gives

$$
\int_{\Omega} d x_{2}\left(\rho_{(2)}^{(\infty)}\left(x_{1}, x_{2}\right)-\rho_{(1)}^{(\infty)}\left(x_{1}\right) \rho_{(1)}^{(\infty)}\left(x_{2}\right)+\delta\left(x_{1}-x_{2}\right) \rho_{(1)}^{(\infty)}\left(x_{2}\right)\right)=\left(z \frac{\partial}{\partial z}\right)^{2} \beta P .
$$

In the theory of fluids, this is referred to as the compressibility sum rule [40].

Proposition 3.1. The limiting continuous determinantal point process specified by Proposition 2.6 satisfies (3.7).

Proof. According to (2.4)

$$
\rho_{(2)}^{(\infty)}(X, Y)-\rho_{(1)}^{(\infty)}(X) \rho_{(1)}^{(\infty)}(Y)=-\left|K^{(\infty)}(X, Y)\right|^{2} .
$$

Substituting (2.35) shows

$$
\int_{-\infty}^{\infty}\left(\rho_{(2)}^{(\infty)}(X, Y)-\rho_{(1)}^{(\infty)}(X) \rho_{(1)}^{(\infty)}(Y)\right) d Y=-z^{2} \int_{-\infty}^{\infty}\left(\frac{\lambda^{(\infty)}(s)}{1+z \lambda^{(\infty)}(s)}\right)^{2} d s .
$$

Substituting (2.34) in the RHS of (3.7) and subtracting $\rho_{(1)}^{(\infty)}(x)$ gives this same expression.

Remark 3.2. 1. The same working, with $\lambda^{(\infty)}(s)$ replaced by $\lambda^{(\epsilon, \infty)}(s)$, verifies that the continuous determinantal point process specified by Proposition 2.8 satisfies (3.7).

2 . In the case of an infinite lattice, the integral over $x_{2}$ should be replaced by a sum over the lattice points, and $\beta P$ on the RHS should be replaced by $\tau \beta P$. Upon this modification, the results of Proposition 2.4 can be checked to be consistent. 
3.2. Gap probabilities. For a point process defined on the real line, let $E^{(\infty)}(n ; J)$ denote the probability that there are exactly $n$ particles within an interval $J$. The case $n=0$ is referred to as the gap probability. Introducing the generating function

$$
\tilde{E}^{(\infty)}(J ; \xi)=\sum_{n=0}^{\infty}(1-\xi)^{n} E^{(\infty)}(n ; J)
$$

it is a standard result (see e.g. $[19, \S 9.1])$ that $\tilde{E}^{(\infty)}(J ; \xi)$ can be written in terms of the correlation functions according to

$$
\tilde{E}^{(\infty)}(J ; \xi)=1+\sum_{j=1}^{\infty} \frac{(-\xi)^{j}}{j !} \int_{J} d x_{1} \cdots \int_{J} d x_{j} \rho_{(j)}^{(\infty)}\left(x_{1}, \ldots, x_{j}\right) .
$$

Specialise now to the case of determinantal correlations

$$
\rho_{(j)}^{(\infty)}\left(x_{1}, \ldots, x_{j}\right)=\operatorname{det}\left[K^{(\infty)}\left(X_{j_{1}}, X_{j_{2}}\right)\right]_{j_{1}, j_{2}=1, \ldots, j} .
$$

Let $\mathbb{K}_{J}$ denote the integral operator supported on $J$ with kernel $K^{(\infty)}(X, Y)$. Then the summation (3.12) can be recognised as a key quantity within the Fredholm theory of integral equations [41], namely the Fredholm determinant,

$$
\tilde{E}^{(\infty)}(J ; \xi)=\operatorname{det}\left(\mathbb{I}-\xi \mathbb{K}_{J}\right)=\prod_{j=0}^{\infty}\left(1-\xi \lambda_{j}^{(J)}\right),
$$

where $\left\{\lambda_{j}^{(J)}\right\}$ denotes the eigenvalues of $\mathbb{K}_{J}$; in fact such a quantity has already appeared in (2.40). Further specialise to the Toeplitz setting so that in $(3.12) K^{(\infty)}\left(X_{j_{1}}, X_{j_{2}}\right)=K^{(\infty)}\left(X_{j_{1}}-X_{j_{2}}, 0\right)$, and introduce the Fourier transform

$$
\hat{K}^{(\infty)}(s)=\int_{-\infty}^{\infty} K^{(\infty)}(x, 0) e^{i s x} d x .
$$

The asymptotic theory of Toeplitz integral operators [42] tells us that for $|J| \rightarrow \infty$,

$$
\tilde{E}^{(\infty)}(J ; \xi) \sim \exp \left(|J| \int_{-\infty}^{\infty} \log \left(1-\xi \hat{K}^{(\infty)}(s)\right) d s\right) .
$$

For the circulant correlation kernels (2.35) we have

$$
\hat{K}^{(\infty)}(s)=\frac{z \lambda^{(\infty)}(s)}{1+z \lambda^{(\infty)}(s)} .
$$

Substituting in (3.15) and comparing with (2.34) shows, upon setting $\xi=1$ and recalling (3.10) that

$$
E^{(\infty)}(0 ; J) \sim e^{-|J| \beta P},
$$

in accordance with the functional form expected for the asymptotic gap probability of a general compressible fluid [43, 44]. 
3.3. Small separation form of $\rho^{(2, \infty)}(X, Y)$. As a rewrite of $(3.8)$ we have

$$
\rho_{(2)}^{(\infty)}(X, Y)=K^{(\infty)}(X, X) K^{(\infty)}(Y, Y)-\left|K^{(\infty)}(X, Y)\right|^{2},
$$

telling us in particular that $\rho_{(2)}^{(\infty)}(X, Y)$ vanishes as $X \rightarrow Y$. Substituting (2.35) in (3.18) and expanding to leading order in $z$ shows

$$
\left.\rho^{(2, \infty)}(X, Y)=z^{2}\left(\left(\int_{-\infty}^{\infty} \lambda^{(\infty)}(s) d s\right)^{2}-\left|\int_{-\infty}^{\infty} e^{2 \pi i s(X-Y)} \lambda^{(\infty)}(s) d s\right|^{2}\right)\right)+O\left(z^{3}\right) .
$$

We see that if $\lambda^{(\infty)}(s)$ decays fast enough at infinity to allow the complex exponential to be expanded to second order, the small distance form of $\rho_{(2)}^{(\infty)}(X, Y)$ will always decay like a quadratic. In fact this will happen without first expanding to low order in $z$.

Proposition 3.3. Let $\lambda^{(\infty)}(s)$ and $\lambda^{(\epsilon, \infty)}(s)$ decay at least as fast as order $1 /|s|^{3}$ at infinity. Then $\rho_{(2)}^{(\infty)}(X, Y)$ goes to zero like $(X-Y)^{2}$ as $X \rightarrow Y$.

Proof. The assumption on the decay of $\lambda^{(\infty)}(s)$ (and its counterpart in the complex Hermitian case) allows the complex exponential in (2.35) to be expanded to second order in $(X-Y)$. In the setting of Proposition 2.6 the term proportional to $(X-Y)$ vanishes due to the parity of the integrand. Furthermore, substituting in the RHS of (3.8) shows that the term independent of $(X-Y)$ also vanishes, leaving the term proportional to $(X-Y)^{2}$ as the leading term. In the setting of Proposition 2.8, applying the same expansion in the analogue of (2.35) we see that the term proportional to $(X-Y)$ no longer vanishes, but nonetheless when substituted in (3.8) its contribution to the expansion of the RHS at this order cancels as does that of the term independent of $(X-Y)$, again leaving the term proportional to $(X-Y)^{2}$ as the leading term.

To leading order in $z$, we see from (3.19) that the behaviour of $\rho^{(2, \infty)}(X, Y)$ will be determined by the behaviour of the Fourier transform of $\lambda^{(\infty)}(s)$. Now from $(2.33)$

$$
\int_{-\infty}^{\infty} \lambda^{(\infty)}(s) e^{-2 \pi i s Y} d s=g(Y)
$$

so we see that the small distance functional form in (3.19) is determined by the rate of vanishing of $g(0)-g(Y)$ as $Y \rightarrow 0$, which for $\lambda^{(\infty)}(s)$ decaying slower that $O\left(1 / s^{3}\right)$ will be slower than of order $Y^{2}$.

3.4. Example of $g(u)$ a Gaussian. Suppose we take for the functional form (2.15) of the matrix elements

Substituting in (2.33) shows

$$
g(u)=\frac{1}{\sqrt{c}} e^{-\pi u^{2} / c}, \quad c>0 .
$$

$$
\lambda^{(\infty)}(s)=e^{-\pi c s^{2}} .
$$

This Gaussian functional form in $s$, decaying faster than any power and being analytic, implies upon repeated integration by parts in (2.35) that $K^{(\infty)}(X, Y)$ decays faster than any inverse power for $|X-Y|$ large, as will be the case whenever $\lambda^{(\infty)}$ has these properties. Specifically for (3.22), this fast decay can be exhibited by first power series expanding in $z$ and then computing the integrals by completing the square to obtain

$$
K^{(\infty)}(X, Y)=-\sum_{p=1}^{\infty} \frac{(-z)^{p}}{\sqrt{c p}} e^{-\pi(Y-X)^{2} / c p}, \quad|z|<1
$$


Thus in fact there is term-by-term Gaussian decay. Parametrise $c$ and $z$ in terms of $\beta$ and $\mu$ according to

$$
c=4 \pi \beta, \quad z=e^{\beta \mu} .
$$

After a simple change of variables in (2.35) the expression (1.9) noted in the Introduction results, which is the correlation kernel for free fermions (or equivalently hard-core bosons) in one-dimension at inverse temperature $\beta$ and chemical potential $\mu$ [45].

Some insight into the relation to free fermions at finite temperature can be obtained by considering $L^{(L)}(X, Y)$ as specified in terms of $g$ by (2.28) in the limit $L \rightarrow \infty$ when it reads $L^{(\infty)}(X, Y)=g(X-Y)$. From (2.39) we then have

$$
p\left(\mathbf{X}_{N}\right) \propto \operatorname{det}\left[L^{(\infty)}\left(X_{j}, X_{k}\right)\right]_{j, k=1}^{N} \propto \operatorname{det}\left[e^{-\pi\left(X_{j}-X_{k}\right)^{2} / c}\right]_{j, k=1}^{N} .
$$

Now replace $\pi / c$ by $1 /\left(2\left(1-q^{2}\right)\right)$ and observe that to leading order

$$
\operatorname{det}\left[e^{-\frac{1}{2} \frac{1}{1-q^{2}}\left(x_{i}-x_{j}\right)^{2}}\right]_{i, j=1}^{N} \underset{q \rightarrow 1}{\sim} \operatorname{det}\left[e^{-\frac{1}{4} \frac{1+q^{2}}{1-q^{2}}\left(x_{i}^{2}+x_{j}^{2}\right)+\frac{q}{1-q^{2}} x_{i} x_{j}}\right]_{i, j=1}^{N} \underset{q \rightarrow 1}{\sim}
$$

as follows by completing the square on the RHS. The significance of this is that it is well known that the RHS of (3.26) can be rewritten in the form of the probability density function for $N$ free fermions in a harmonic well on a line, in equilibrium at a finite temperature; see [46, §1] for a clear derivation. After appropriate scaling, the bulk correlation kernel is precisely (1.9) [47-50].

It is instructive to consider the $\beta \rightarrow \infty$ limit of (1.9). We see

$$
\left.K^{(\infty)}(X, Y)\right|_{\beta \rightarrow \infty}=\frac{1}{2 \pi} \int_{-\sqrt{\mu}}^{\sqrt{\mu}} e^{i(Y-X) k} d k=\frac{\sin \sqrt{\mu}(Y-X)}{\pi(Y-X)} .
$$

Setting $X=Y$ gives for the particle density the value $\rho_{(1)}^{(\infty)}=\sqrt{\mu} / \pi$. Taking this to equal unity as a normalisation, we then recognise (3.27) as the sine kernel from random matrix theory as applies to the bulk of unitary invariant ensembles (see e.g. [19, Ch. 7]), the latter having a well known analogy with the ground state of free fermions on a line (see e.g. [51]).

In relation to the gap probabilities associated with (1.9), from (3.13) these are determined by the eigenvalues of $\mathbb{K}_{J}$. With $J=(-x, x)$, in the present setting these satisfy

$$
\int_{-x}^{x} K^{(\infty)}(X-Y, 0) f_{j}(Y) d Y=\lambda_{j}^{(J)} f_{j}(X),
$$

where $\left\{f_{j}(X)\right\}$ are the corresponding eigenfunctions. Taking the Fourier transform of both sides with respect to $X$, and also writing $f_{j}(X)$ in terms of its Fourier transform, this can be rewritten

$$
\hat{K}^{(\infty)}(k) \int_{-\infty}^{\infty} \frac{\sin (x(k-s))}{\pi(k-s)} \hat{f}_{j}(s) d s=\lambda_{j}^{(J)} \hat{f}_{j}(k) .
$$

Consider this equation with

$$
\hat{f}_{j}(k) \mapsto\left(\hat{K}^{(\infty)}(k)\right)^{1 / 2} \hat{f}_{j}(k),
$$

and read off the explicit form of $\hat{K}^{(\infty)}(k)$ from (1.9). This tells us that we have the identity [52]

$$
\prod_{j=0}^{\infty}\left(1-\xi \lambda_{j}^{(J)}\right)=\prod_{j=0}^{\infty}\left(1-\xi \tilde{\lambda}_{j}^{(J)}\right),
$$

where $\left\{\tilde{\lambda}_{j}^{(J)}\right\}$ are the eigenvalues of the integral operator on all of $\mathbb{R}$ with kernel

$$
\tilde{K}^{(\infty)}(k, s)=\left(\frac{1}{e^{\beta\left(k^{2}-\mu\right)}+1}\right)^{1 / 2} \frac{\sin (x(k-s))}{\pi(k-s)}\left(\frac{1}{e^{\beta\left(s^{2}-\mu\right)}+1}\right)^{1 / 2} .
$$


The significance of the functional form (3.32), in contrast to (1.9), is that the former has the structure of a so-called integrable kernel [53]. Associated with integral kernels are differential equations. Explicitly, with $(3.31)$ denoted by $\Delta(x, \mu, \xi)$, we have that $\sigma(x, t, \xi):=\log \Delta(\sqrt{\beta} x, t / \beta, \xi)$ satisfies the partial differential equation [52]

$$
\left(\partial_{t} \partial_{x}^{2} \sigma\right)^{2}=-4\left(\partial_{x}^{2} \sigma\right)\left(2 x \partial_{t} \partial_{x} \sigma+\left(\partial_{t} \partial_{x} \sigma\right)^{2}-2 \partial_{t} \sigma\right)
$$

subject to the small- $x$ expansion

$$
\sigma(x, t, \xi)=-\frac{\xi}{\pi}\left(\int_{-\infty}^{\infty} \frac{d \lambda}{1+e^{\lambda^{2}-t}}\right) x-\frac{\xi^{2}}{2 \pi^{2}}\left(\int_{-\infty}^{\infty} \frac{d \lambda}{1+e^{\lambda^{2}-t}}\right)^{2} x^{2}+\cdots
$$

Consider now the gap probability in the $\beta \rightarrow \infty$ limit. We know from (3.27) that

$$
\left.\lim _{\beta \rightarrow \infty}(\sqrt{t} / \beta) \tilde{K}^{(\infty)}(\sqrt{t} k / \beta, \sqrt{t} s / \beta)\right|_{\substack{x \mapsto \sqrt{\beta} x \\ \mu=t / \beta}}=\frac{\sin (\sqrt{t} x(k-s))}{\pi(k-s)},
$$

supported on $k, s \in[-1,1]$. Thus in this limit $\sigma(x, t, \xi)$ depends on $x, t$ only through quantity $\tau:=\sqrt{t} x$ and moreover

$$
\sigma(x, t, \xi) \rightarrow \log \operatorname{det}\left(\mathbb{I}-\xi \mathbb{K}_{(-1,1)}^{(\tau, \text { sine })}\right)=: \log \Delta(\tau, \xi),
$$

where $\left.\mathbb{K}_{(-1,1)}^{(\tau, \text { sine })}\right)$ denotes the integral operator supported on $(-1,1)$ with kernel $(3.27)$ and $\sqrt{\mu}=\tau$. As noted in [53], with

$$
\sigma_{0}(\tau, \xi):=\tau \partial_{\tau} \log \Delta(\tau, \xi)
$$

it follows from (3.33), with prime denoting differentiation with respect to $\tau$, that

$$
\left(\tau \sigma_{0}^{\prime \prime}\right)^{2}=-4\left(\tau \sigma_{0}^{\prime}-\sigma_{0}\right)\left(4 \tau \sigma_{0}^{\prime}+\left(\sigma_{0}^{\prime}\right)^{2}-4 \sigma_{0}\right)
$$

This nonlinear second order differential equation, which relates to the Hamiltonian theory of the Painlevé V system, was obtained for the Fredholm determinant for the sine kernel in (3.36) by the Kyoto school [54]; see also [19, §8.3.5].

3.5. Example of $h(u)=1 / u$. Before specialising Proposition 2.8, it is of interest to make note of the evaluation of the determinant in (2.38) which holds for this choice of $h$. This requires use of the Cauchy double alternant identity (see e.g. [55])

$$
\operatorname{det}\left[\frac{1}{x_{j}-y_{k}}\right]_{j, k=1}^{N}=\frac{\prod_{1 \leq j<k \leq N}\left(x_{j}-x_{k}\right)\left(y_{k}-y_{j}\right)}{\prod_{j, k=1}^{N}\left(x_{j}-y_{k}\right)}
$$

Noting that with

we have

$$
x_{j}=\frac{1}{2 i} e^{2 \pi i X_{j} / L} e^{-2 \pi \epsilon / L}, \quad y_{j}=\frac{1}{2 i} e^{2 \pi i X_{j} / L} e^{2 \pi \epsilon / L},
$$

$$
\operatorname{det}\left[\frac{\pi}{L \sin \left(\pi\left(X_{j}-X_{k}+2 i \epsilon\right) / L\right)}\right]_{j, k=1}^{N}=\left(\frac{\pi}{L}\right)^{N} \prod_{j=1}^{N} e^{-2 \pi i N X_{j} / L} \operatorname{det}\left[\frac{1}{x_{j}-y_{k}}\right]_{j, k=1}^{N},
$$

application of (3.41) gives for $h(u)=i / u$,

$$
\operatorname{det}\left[L\left(X_{j}, X_{k}\right)\right]_{j, k=1}^{N}=\left(\frac{\pi i}{L}\right)^{N} \frac{\prod_{1 \leq j<k \leq N}\left(\sin \left(\pi\left(X_{k}-X_{j}\right) / L\right)\right)^{2}}{\prod_{j, k=1}^{N} \sin \left(\pi\left(X_{j}-X_{k}+2 i \epsilon\right) / L\right)} .
$$

First considered in [6] (see also [33]), (3.42) corresponds to the Boltzmann factor for a statistical mechanical system of $N$ particles (two-dimensional charges interacting via a logarithmic potential) in equilibrium at inverse temperature $\beta=2$, confined to the interval $[0, L]$ on the $x$-axis in periodic 
boundary conditions and in the presence of a perfect conductor at $y=\epsilon$. For each charge at $(X, 0)$, the perfect conductor creates an image charge of opposite sign at $(X, 2 \epsilon)$. We remark that in the reference [33], the PDF corresponding to (3.42) is related to the theory of parametric eigenvalue motion due to Pechukas [56] and Yukawa [57], as discussed extensively in the book on quantum chaos by Haake [58].

Returning now to Proposition 2.8, setting $h(u)=1 / u$ in (2.46) gives

$$
\lambda^{(\epsilon, \infty)}(s):=i \int_{-\infty}^{\infty} h(-Y+2 i \epsilon) e^{2 \pi i s Y} d Y=2 \pi \begin{cases}e^{-4 \pi \epsilon s}, & s \geq 0 \\ 0, & \text { otherwise }\end{cases}
$$

Substituting this for $\lambda^{(\infty)}(s)$ in (2.34) and (2.35) specifies the corresponding pressure and correlation kernel. Specifically, for the latter [6]

$$
K^{(\epsilon, \infty)}(X, Y)=\int_{0}^{\infty} \frac{e^{2 \pi i(X-Y) s}}{(1 / 2 \pi z) e^{4 \pi \epsilon s}+1} d s
$$

From the viewpoint of universal forms for two-point correlations in Coulomb systems of restricted dimension [59], of relevance is the large $X$ asymptotic form of (3.44), which is uniform for large $\epsilon$, when furthermore $z$ is related to $\epsilon$ by

$$
(1 / 2 \pi z)=e^{-4 \epsilon h}, \quad h>0 .
$$

Integrating by parts once, then extending the domain of integration to all of $\mathbb{R}$ in the resulting integral gives

$$
K^{(\epsilon, \infty)}(X, Y) \underset{|X-Y| \rightarrow \infty}{\sim} \frac{1}{2 \pi i}\left(-\frac{1}{X-Y}-\frac{e^{2 i h(X-Y)}}{(2 \epsilon / \pi) \sinh (\pi(X-Y) / 2 \epsilon)}\right) .
$$

This substituted in (3.8) implies

$$
\rho^{(2, \infty)}(X, Y)-\left(\rho^{(1, \infty)}\right)^{2} \underset{|X-Y| \rightarrow \infty}{\dot{\sim}}-\frac{1}{4 \pi^{2}(X-Y)^{2}}-\frac{1}{8 \epsilon^{2} \sinh ^{2} \pi(X-Y) / 2 \epsilon} .
$$

Here the modification of the asymptotic symbol $\dot{\sim}$ indicates that oscillatory terms averaging to zero are ignored. This is the universal form predicted in [59, Eq. (3.4)].

Write now relate $z$ to $\epsilon$ by (3.45) and take the limit $\epsilon \rightarrow \infty$ with $X, Y$ fixed. We see that

$$
\left.K^{(\epsilon, \infty)}(X, Y)\right|_{\substack{(1 / 2 \pi z)=e^{-2 \epsilon h / \pi} \\ \epsilon \rightarrow \infty}}=e^{i(X-Y) h} \frac{\sin (h(X-Y))}{\pi(X-Y)} .
$$

Substituting in the determinant formula (3.10) we see that the contributions from the factors of the form $e^{i(X-Y) h}$ cancel and as in (3.27) the sine kernel from random matrix theory is reclaimed, as already known from [6].

Remark 3.4. Although not considered further in the present work, we note that the choice $h(u)=$ $1 / \sinh u$ is also of interest from the viewpoint of the study [59]. Moreover, as observed in that latter reference, in the limit $L \rightarrow \infty$, an identity analogous to (3.42) holds true, showing that their is an underlying pair potential. 
3.6. Higher dimensions. The appropriate generalisation of the circulant $L$-ensemble structure to higher dimensions - say to a particle system confined to a cube $[0, L]^{d}$ with periodic boundary conditions - is to define vectors $\mathbf{x}=\left(x_{1}, \ldots, x_{d}\right)$ (and similarly $\mathbf{y}$ ) and extend the definition (2.28) to read

$$
L^{(L)}(\mathbf{x}, \mathbf{y})=g\left((L / \pi) \sin \left(\pi\left(x_{1}-y_{1}\right) / L\right), \ldots,(L / \pi) \sin \left(\pi\left(x_{d}-y_{d}\right) / L\right)\right) .
$$

With this done, the probability density function for a configuration $\mathbf{X}_{N}$ in the cube is again given by (2.39), but with $\mathbb{L}$ in (2.40) now specified on the interval $[-L / 2, L / 2]^{d}$ with kernel (3.49),

$$
\mathbb{L}[f](\mathbf{x})=\int_{[-L / 2, L / 2]^{d}} g\left((L / \pi) \sin \left(\pi\left(x_{1}-y_{1}\right) / L\right), \ldots,(L / \pi) \sin \left(\pi\left(x_{d}-y_{d}\right) / L\right)\right) f(\mathbf{y}) d \mathbf{y} .
$$

The normalised eigenfunctions are

$$
v_{\mathbf{p}}(\mathbf{x})=\frac{1}{L^{d / 2}} \prod_{j=1}^{d} e^{2 \pi i p_{j} x_{j} / L}=\frac{1}{L^{d / 2}} e^{2 \pi i \mathbf{p} \cdot \mathbf{x}}, \quad p_{j} \in \mathbb{Z}(j=1, \ldots, d) .
$$

Using the multidimensional analogues of (2.41) and (2.42), the results of Proposition 2.6 can be extended to higher dimensions.

Proposition 3.5. Consider the d-dimensional determinantal point-process of the L-ensemble type specified by (3.49) and surrounding text. Let

$$
\lambda^{(d, \infty)}(\mathbf{s})=\int_{\mathbb{R}^{d}} g(\mathbf{t}) e^{2 \pi i \mathbf{s} \cdot \mathbf{t}} d \boldsymbol{t} .
$$

We have

$$
\beta P=\int_{\mathbb{R}^{d}} \log \left(1+z \lambda^{(d, \infty)}(\mathbf{s})\right) d \mathbf{s},
$$

and

$$
K^{(d, \infty)}(\mathbf{x}, \mathbf{y})=z \int_{\mathbb{R}^{d}} \frac{e^{2 \pi i(\mathbf{y}-\mathbf{x}) \cdot \mathbf{s}} \lambda^{(d, \infty)}(\mathbf{s})}{1+z \lambda^{(d, \infty)}(\mathbf{s})} d \mathbf{s} .
$$

As an explicit example, consider the $d$-dimensional generalisation of Gaussian (3.21),

$$
g(\mathbf{u})=\frac{1}{c^{d / 2}} e^{-\pi \mathbf{u}^{2} / c}, \quad c>0 .
$$

Substituting in (3.52) gives

$$
\lambda^{(d, \infty)}(\mathbf{s})=e^{-\pi c \mathbf{s}^{2}} .
$$

The formula (3.53) for the pressure, from the spherical symmetry of the integrand, then simplifies upon the use of polar coordinates to read

$$
\beta P=\left|\Omega_{d}\right| \int_{0}^{\infty} r^{d-1} \log \left(1+z e^{-\pi c r^{2}}\right) d r
$$

where $\left|\Omega_{d}\right|$ denotes the surface area of the unit ball in $d$-dimensions, while the formula (3.54) for the correlation kernel reads

$$
K^{(d, \infty)}(\mathbf{x}, \mathbf{y})=\left(\frac{1}{2 \pi}\right)^{d} \int_{\mathbb{R}^{d}} \frac{e^{i(\mathbf{y}-\mathbf{x}) \cdot \mathbf{k}}}{e^{\beta\left(\mathbf{k}^{2}-\mu\right)}+1} d \mathbf{k} .
$$

In (3.58) the parameters $\beta$ and $\mu$ have been introduced in favour of $c$ and $z$ as in (1.9). The resulting expression can be recognised as the correlation kernel for free fermions in $d$-dimensions in equilibrium at inverse temperature $\beta$ and chemical potential $\mu$ (see [50], where it is furthermore noted that the introduction of polar coordinates can be used to reduce (3.58) down to a onedimensional integral involving a Bessel function). 
A question of interest is the asymptotic form of the probability that there are no particles in a region $\Lambda$ say of $\mathbb{R}^{d}$ — what was termed in the one-dimensional case in Section $\S 3.2$ as the gap probability, but what in higher dimensions is usually referred to as the hole probability. The fact that $K^{(d, \infty)}(\mathbf{x}, \mathbf{y})$ only depends on the differences of the components allows for the determination of the limiting asymptotic form for $|\Lambda| \rightarrow \infty$ [60]

$$
E(0, \Lambda) \sim e^{-|\Lambda| \beta P}
$$

where $\beta P$ is given by (3.57), in keeping with (3.17). Generally this asymptotic behaviour of the hole probability is expected whenever the particle system is compressible [43]. Note that the latter condition ceases to hold in the zero temperature, $\beta \rightarrow \infty$, limit of (3.58). For results on the corresponding asymptotic form of the hole probability, see the recent work [61].

Taken literally the complex Hermitian circulant matrix construction (2.38) does not have a generalisation to higher dimension due to the use of the complex unit $i$ as effectively extending from the real line to the $x y$-plane. However, by extending the $L$-ensemble formalism from one to two-components, a two-dimensional complex Hermitian Toeplitz construction is known from the work of Gaudin on the two-dimensional two-component Coulomb gas at a special coupling [7, 13].

Afterword. My earliest memory of encountering the work of F. Haake has through his work with Grobe and Sommers on the hole probability in the Ginibre ensemble [62]. I was able to use this to deduce the first four terms in its asymptotic expansion [63]. Around the same time the first edition of F. Haake's celebrated book Quantum signatures of chaos [58] appeared. In addition to be taken by the discussion relating the Ginibre ensemble to dissipative quantum systems, I payed particular attention to the sections on the so-called Pechukas-Yukawa gas, and Dyson's Brownianmotion model, in the chapter on Level Dynamics. The latter was very influential in shaping my own subsequent work on the topic [19, Ch. 11].

Acknowledgements. This research is part of the program of study supported by the Australian Research Council Centre of Excellence ACEMS, and the project DP210102887. The presentation has benefitted from a number of considered remarks put forward by the referees.

\section{REFERENCES}

[1] K. Husimi, Some formal properties of the density matrix, Proc. Phys. Math. Soc. Jpn. 22 (1940), 264-314 .

[2] O. Macchi. The coincidence approach to stochastic point processes, Adv. Appl. Probab., 7 (1975), 83-122.

[3] F.J. Dyson, Statistical theory of energy levels of complex systems III, J. Math. Phys. 3 (1962), $166-175$.

[4] J. Ginibre, Statistical ensembles of complex, quaternion, and real matrices, J. Math. Phys. 6 (1965), 440-449.

[5] A. Alastuey and B. Jancovici, On the two-dimensional one-component Coulomb plasma, J. Physique 42 (1981), 1-12.

[6] M. Gaudin, Une famille à un paramètre d'ensembles unitaires, Nucl. Phys. 85 (1966), 545-575.

[7] M. Gaudin, L'isotherme critique d'un plasma sur réseau ( $\beta=2, d=2, n=2)$, J. Physique 46 (1985), 1027-1042. 
[8] P.W. Kasteleyn, Graph theory and crystal physics, In "Graph Theory and Theoretical Physics", pgs. 43-110, Academic Press, London, 1967.

[9] F.J. Dyson, A Brownian motion model for the eigenvalues of a random matrix, J. Math. Phys. 3 (1962), 1191-1198.

[10] M.E. Fisher, Walks, walls, wetting, and melting, J. Stat. Phys. 34 (1984), 667-729.

[11] E. Lieb and D.C. Mattis, Mathematical physics in one dimension, Academic Press, NewYork, 1966.

[12] M.L. Mehta, Random matrices, 2nd ed., Academic Press, New York, 1991.

[13] P.J. Forrester, Exact results for two-dimensional Coulomb systems, Phys. Reports 301 (1998), $235-270$.

[14] A. Borodin and G. Olshanski, Distributions on partitions, point processes and the hypergeometric kernel, Comm. Math. Phys. 211 (2000), 335-358.

[15] K. Johansson, Non-intersecting paths, random tilings and random matrices, Prob. Theory Related Fields 123 (2002), 225-280.

[16] N.M. Bogoliubov, XX Heisenberg chain and random walks, J. Math. Sci. 138 (2006), 56365643.

[17] J.B. Hough, M. Krishnapur, Y. Peres, and B. Virág, Zeros of Gaussian analytic functions and determinantal point processes, American Mathematical Society, Providence, RI, 2009.

[18] S. Torquato and A. Scardicchio and C.E. Zachary, Point processes in arbitrary dimension from fermionic gases, random matrix theory, and number theory, J. Stat. Mech. (2008), P110019.

[19] P.J. Forrester, Log-gases and random matrices, Princeton University Press, Princeton, NJ, 2010.

[20] M. Katori, Bessel processes, Schramm-Loewner evolution, and the Dyson model, Springer briefs in mathematical physics, vol. 11, Springer, Berlin, 2016.

[21] D.S. Dean, P. Le Doussal, S.N. Majumdar and G. Schehr, Non-interacting fermions in a trap and random matrix theory, J. Phys. A 52, (2019), 144006.

[22] A. Soshnikov, Determinantal random point fields, Russian Math. Surveys 55 (2000), 923-975.

[23] D. J. Daley and D. Vere-Jones, An introduction to the theory of point processes Vol. I. SpringerVerlag, New York, second edition, 2003. Elementary theory and methods.

[24] R. Lyons, Determinantal probability measures, Publ. Math. Inst. Hautes Etudes Sci., 98 (2003), 167-212.

[25] T. Shirai and Y. Takahashi, Random point fields associated with certain Fredholm determinants. I. Fermion, Poisson and boson point processes, J. Funct. Anal., 205 (2003) 414-463.

[26] A. Borodin, P. Diaconis, and J. Fulman, On adding a list of numbers (and other one-dependent determinantal processes), Bull. Am. Math. Soc. 47 (2010), 639-670.

[27] A. Borodin, Determinantal point processes, The Oxford Handbook of Random Matrix Theory (G. Akemann, J. Baik, and P. di Francesco, eds.), Oxford University Press, Oxford, 2011, pp. 231-249.

[28] A. Kulesza and B. Taskar, Determinantal point processes for machine learning, Found. Trends Mach. Learn. 5 (2012), 123-286.

[29] F. Lavancier, J. Møller, and E. Rubak, Determinantal point process models and statistical inference, J. Royal Stat. Soc.: Series B 77 (2015), 853-877. 
[30] L. Pastur and M. Shcherbina, Eigenvalue distribution of large random matrices, American Mathematical Society, Providence, RI, 2011.

[31] A.I. Bufetov and Y. Qiu, Determinantal point processes associated with Hilbert spaces of holomorphic functions, Commun. Math. Phys. 351 (2017), 1-44.

[32] M. Katori and T. Shirai, Partial isometries, duality, and determinantal point processes, arXiv:1903.04945.

[33] P.J. Forrester, Statistical properties of the eigenvalue motion of Hermitian matrices, Phys. Lett. A 173 (1993), 355-359.

[34] E. I. Broman, One-dependent trigonometric determinantal processes are two-block-factors, Ann. Probab. 33 (2005), 601-609.

[35] H. Dai, Z. Geary and L.P. Kadanoff Asymptotics of eigenvalues and eigenvectors of Toeplitz matrices, J. Stat. Mech. , 2009 (2009), P05012.

[36] T. Mondal, S. Sadhukhan, and P. Shukla, Extended states with Poisson spectral statistics, Phys. Rev. E 95, (2017), 062102.

[37] A. Bose, Patterned Random matrices. Chapman and Hall/CRC, Boca Raton, FL, 2018.

[38] T. Mondal and P.Shukla, Statistical analysis of chiral structured ensembles: Role of matrix constraints, Phys. Rev. E 99 (2019), 022124.

[39] E. Bogomolny, Spectral statistics of random Toeplitz matrices, Phys. Rev. E 102 (2020), 04101(R).

[40] J.-P. Hansen and I.R. McDonald, Theory of Simple Liquids, Academic Press, 3rd Edition, 2006.

[41] E.T. Whittaker and G.N. Watson, A course of modern analysis, 4th ed., Cambridge University Press, Cambridge, 1927.

[42] M. Kac, Toeplitz matrices, translation kernels, and a related problem in probability theory, Duke Math. J. 21 (1954), 501-509.

[43] P.J. Forrester and C. Pisani, The hole probability in log-gas and random matrix systems, Nucl. Phys. B 374 (1992), 720-740.

[44] P.J. Forrester, Log-gases, random matrices and the Fisher-Hartwig conjecture, J. Phys. A 26 (1993), 1179-1192.

[45] A. Lenard, One-dimensional impenetrable bosons in thermal equilibrium, J. Math. Phys. 7 (1966), 1268-1272.

[46] K. Liechty and D. Wang, Asymptotics of free fermions in a quadratic well at finite temperature and the Moshe-Neuberger-Shapiro random matrix model, Ann. Inst. H. Poincaré Probab. Statist. 56 (2020), 1072-1098.

[47] M. Moshe, H. Neuberger, and B. Shapiro, Generalized ensemble of random matrices, Phys. Rev. Lett. 73 (1994), 1497-1500.

[48] A. M. Garcia-Garcia and J. J. M. Verbaarschot, Critical statistics in quantum chaos and Calogero-Sutherland model at finite temperature, Phys. Rev. E 67, (2003) 046104.

[49] K. Johansson, From Gumbel to Tracy-Widom, Prob. Theor. Rel. Fields 138 (2007), 75-112.

[50] D.S. Dean, P. Le Doussal, S.N. Majumdar and G. Schehr, Non-interacting fermions at finite temperature in a d-dimensional trap: universal correlations, Phys. Rev. A 94, (2016), 063622.

[51] D.S. Dean, P. Le Doussal, S.N. Majumdar and G. Schehr, Non-interacting fermions in a trap and random matrix theory, J. Phys. A 52, (2019), 144006. 
[52] A.R. Its,, A.G. Izergin, and V. E. Korepin. Temperature correlators of the impenetrable Bose gas as an integrable system, Commun. Math. Phys. 129 (1990), 205-222.

[53] A.R. Its, A.G. Izergin, V.E. Korepin, and N.A. Slavnov, Differential equations for quantum correlation functions, Int. J. Mod. Phys B 4 (1990), 1003-1037.

[54] M. Jimbo, T. Miwa, Y. Môri, and M. Sato, Density matrix of an impenetrable Bose gas and the fifth Painlevé transcendent, Physica 1D (1980), 80-158.

[55] A.C. Aitken, Determinants and matrices, 9th ed., Oliver and Boyd, Edinburgh \& London, 1956.

[56] P. Pechukas, Distribution of energy eigenvalues in the irregular spectrum, Phys. Rev. Lett., 51 (1983), 943-946.

[57] T. Yukawa, Lax form of the quantum eigenvalue problem, Phys. Lett. A 116 (1986) 227-230.

[58] F. Haake, Quantum signatures of chaos, Springer, Berlin, 1992.

[59] P.J. Forrester, B. Jancovici, and G. Téllez, Universality in some classical Coulomb systems of restricted domain, J. Stat. Phys. 84 (1996), 359-378.

[60] H. Widom, A theorem on translation kernels in $n$ dimensions, Trans. Amer. Math. Soc. 94 (1960), 170-180.

[61] G. Gouraud, P. Le Doussal, and G. Schehr, Hole probability for noninteracting fermions in a d-dimensional trap, arXiv:2104.08574.

[62] R. Grobe, F. Haake, and H.-J. Sommers, Quantum distinction of regular and chaotic dissipative motion, Phys. Rev. Lett.61(1988) 1899.

[63] P.J. Forrester, Some statistical properties of the eigenvalues of complex random matrices, Phys. Lett. A, 169, 21-24.

School of Mathematical and Statistics, ARC Centre of Excellence for Mathematical and Sta-

tistical Frontiers, The University of Melbourne, Victoria 3010, Australia

Email address: pjforr@unimelb.edu.au 